\title{
Coordinator Role Mobility Method for Increasing the Life Expectancy of Wireless Sensor Networks
}

\author{
Aleksejs Jurenoks ${ }^{1}$, Dejan Jokićb ${ }^{2}$ \\ ${ }^{1}$ Faculty of Computer Science and Information Technology, Riga Technical University, Latvia, \\ ${ }^{2}$ University of East Sarajevo, Bosnia and Herzegovina
}

\begin{abstract}
The general problem of wireless sensor network nodes is the low-power batteries that significantly limit the life expectancy of a network. Nowadays the technical solutions related to energy resource management are being rapidly developed and integrated into the daily lives of people. The energy resource management systems use sensor networks for receiving and processing information during the realia time. The present paper proposes using a coordinator role mobility method for controlling the routing processes for energy balancing in nodes, which provides dynamic network reconfiguration possibilities. The method is designed to operate fully in the background and can be integrated into any exiting working system.
\end{abstract}

Keywords - Industry 4.0, life expectancy, sensor network.

\section{INTRODUCTION}

The number of studies related to wireless sensor network liftime expectancy is increasing daily. Lately, the number of systems that do not require human intervention in the system process has been increasing (machine-to-machine communication).

Low power computing systems are regaining more and more popularity. Nowadays the technology makes it possible to reduce the size of existing computing systems resulting in a low-cost low power autonomous system with the ability to use both physical and wireless networks for transmitting information. The greatest popularity this type of technology has gained for receiving information from sensors and in the area of control systems. Networks that use low power computing systems and transmit data via wireless connectivity are known as wireless sensor networks and their main task is to receive information from sensors and transmit it through the network.

Sensor networks are primarily intended for the collection of information [4]. This means that in any network there are terminal nodes for collection of information, the router nodes for transmission of information, and one or more coordinator nodes on which all the collected information is sent across the network. The nodes pertaining to information storage and processing are mainly equipped with an uninterrupted power supply, independent distribution network connectivity and high performance computing system. This means that the direction of the data is definitely known in the sensor network, the information from the data collectors (terminals) is sent to the information storage and processing nodes by using existing routers of the network. The capacity of data traffic near coordinator node is much higher than in the distant points [7].

The differentiated energy consumption in hubs [8], [9] always appears in the operating sensor network; as a result, the existing elements close to processing nodes faster than others stop operating due to a lack of electricity and the network ceases its overall work.

\section{RELATED WORK}

The recent research related to wireless sensor networks can be divided into two directions:

- Studies referring to modelling and optimising the data flow. It needs to be emphasised here that the sensor network traffic transmission depends on the use of a network and the performance scenario. There are systems where the frequency and quantity of the conveyed information are strictly determined and there is no room for network optimisation on display; therefore, irregular consumption of energy appears in the network nodes.

- Studies related to the improvement of technical characteristics of a wireless sensor network for an increase in the life expectancy of a network. The main drawback of the wireless sensor network is the lowpower batteries, which significantly limit the lifetime of the network. Currently, there are a number of methods that allow solving this problem. These include the choice of the capacity of an individual battery, the density of node deployment, the adjustment of transmitter power, the application of the energyefficient data transfer protocol, the positioning of network nodes and other methods that are associated with implementing additional network costs.

A new method has relatively recently been proposed for balancing energy through the mobility of the network coordinator node, which provides the opportunity of reconfiguring the dynamic network or, in other words, the change of network topology.

\section{Methods FOR ROUTE CREATION FOR DATA TRANSMISSION IN THE NETWORK}

In previous publication [3], it was stated that wireless sensor networks could be represented with the help of a directed graph. Based on the structure of the graph, each route $(v, u)$ can be used for transmitting information from node $v$ to node $u$. Each of the $v$ nodes has a defined number of adjacent 
objects $N(v) \subset V$ that may communicate with the node $v$. Whilst generating a route, the broadband data retransmission is used in the network [5], and the information is transmitted from the node $N(v)$ to the entire network simultaneously.

The following data flow handling algorithm in the network is described in the article. After receiving the transmitted information from node $S$, which is designed to receive node $D$, node $V$ uses the following sequence of actions:

- The potential transponder set $R(v, d) \subset N_{v}$ is selected and sorted by priority from the set $N(v)$.

- The potential transponder set of nodes belongs to the set $R(v, d) \in N_{v}$ and determines the operating strategy of node $\mathrm{v}$.

- Information on the functionality of the node is recorded in the service of the transmission packet and distributed to the network management agent.

To determine the retransmission priority, it is important to take into account the remaining amount of energy in each individual node. The node control module selects a transponder with the greatest amount of remaining energy $E_{0}$ in the node. The route building algorithm completely concludes its operations once it has reached the recipient node $d$.

The paper states that each network element can operate in two modes (terminal or router). In order to split the task into the early stage of network, reconfiguration of each node has to remain in standby mode. During the standby mode the remaining amount of energy $E_{0}^{n}$ is assessed in the node.

The route of the wireless sensor network from the terminal $S$ to the coordinator $D-S(s, d)$ takes the dendriform structure, which is built using the recursive approach from terminal to the coordinator. The first stage is defined by the possible network router set $R(s, d) \in N(s)$. This will allow describing the path $S(s, d)$ as the sorted sub-route list:

$$
S(s, d)=\left[S\left(v_{1}, d\right), \ldots, S\left(v_{n}, d\right)\right], v_{j} \in R(s, d) .
$$

The next step is to set up the local action strategy $R\left(v_{j}, d\right)$ for each router. As a result, each list of sub-routes can be improved:

$S\left(v_{j}, d\right)=\left[S\left(v_{j, 1}, d\right), \ldots, S\left(v_{j}, m, d\right)\right], v_{j, i} \in R\left(v_{j}, d\right)$.

The path from the terminal to the coordinator can be displayed as the dendriform list placed on the second level:

$$
\begin{aligned}
& S(s, d) \\
& =\left[\left[S\left(v_{1,1}, d\right), \ldots, S\left(v_{1, m 1}, d\right)\right], \ldots,\left[S\left(v_{n, 1}, d\right), \ldots, S\left(v_{n, m_{n}}, d\right)\right]\right] .
\end{aligned}
$$

When creating a route, the level is extended until the router list contains only one coordinator $D$ node. In this case, the segment of the sensor network is equipped with the only remaining coordinator node. Henceforth, it is to be assumed that the recipient node $D$ (the coordinator) is fixed and will not be used in any formulas.

Let us introduce a way to combine paths. Let us suppose that $S(v)$ is the path from the node $V$ to the coordinator in the router list $R(v)=\left(v_{1}, v_{2}, \ldots, v_{n}\right)$ where the node $U$ does not exist. Supposedly, $S(u)$ is a path consisting of nodes $R(v)$ that have been excluded from the list.

Let us define the path that uses the combined list of $S(v) \in$ $S(u)$ where nodes $U$ are used with low levels of a factor, like this: $R(v)=\left(v_{1}, v_{2}, \ldots, v_{n}, u\right)$.

\section{A. Using Metrics to Determine the Use of the Path}

It can be assumed that the number of paths leading to the node $D$ has been assigned with the value of metrics $l(S(s))$, which is found in the indexed amount and can be compared. Metrics in similar models are built using the recursive approach from top to bottom defining the conditions for establishing the route [11].

$l\left(S(v)=F\left(l\left(S\left(v_{1}\right), l\left(S\left(v_{2}\right), \ldots, l\left(S\left(v_{n}\right)\right), v_{j} \in R(v)\right.\right.\right.\right.$.

For the routing algorithm of a wireless sensor network to be optimal, the management module of the node executes three validations:

\section{Defining the importance of routers}

The node $U$ is useful to the node $V$, if the following condition is fulfilled:

$$
\left(\begin{array}{c}
S\left(v_{1}\right) \\
S\left(v_{2}\right) \\
\ldots \\
S\left(v_{n}\right)
\end{array}\right)_{T} \underset{t, \text { rauž }}{\longrightarrow}\left(\begin{array}{c}
S\left(v_{i_{1}}\right) \\
S\left(v_{i_{2}}\right) \\
\ldots \\
S\left(v_{i_{n}}\right)
\end{array}\right)_{T}
$$

where $S\left(v_{i_{j}}\right)>S\left(v_{i_{j+1}}\right), j=1,2, \ldots, n-1$.

This condition serves a significant purpose. From the list of metrics $S(v)$, all metrics whose indications are low will be selected. Correspondingly, the possibility to entrust the role of the router to the node will be ruled out with a small amount of power left.

\section{Preserving the priorities}

The metrics stay at the same level of priority if $l(S(u)) \leq$ $l(S(v))$ is derived from $l(S(u) \leq l(S(v) \oplus S(u))$ for all $\forall v \in$ $V$ and $\forall u \in N(v)$. This means that before adding a router to the $U$ list $S(v)$ it is assessed whether it will be better than anything else from the list $S(v)$.

\section{The determination of the optimality of routers}

It determines the behaviour of metrics during full reconfiguration of the router list. Let us assume that $R(v)=$ $\left(v_{1}, v_{2}, \ldots, v_{n}\right)$ is the number of routers for the node $V$. Let us introduce an additional list $R^{*}(v)$ with nodes that are sorted by metrics in an ascending order $-l\left(S\left(v_{n}\right)\right) \leq l\left(S\left(v_{n+1}\right)\right)$. The list of ordered metrics will allow for the choice of optimal number of routers for the node $V$ :

$$
l\left[S_{R *(v)}(v)\right] \leq\left[S_{R *(v)(v)}\right]
$$




\section{B. Path Selection Algorithm}

The procedure Breadth-Search is considered the basis of the classic routing algorithm and it allows analysing the graph $G$ $(V, E)$ in width [10]. During the procedure, two variables are added to each network node $\operatorname{dist}(v)$ that indicate the value of the optimum distance length from the node $V$ to the coordinator and $\operatorname{pred}(v)$, which indicates the length of path from node $V$ to the closest router. There is a definite starting value of the variable $\operatorname{dist}(v) \rightarrow \infty$. This means that the node has not been visited yet. The coordinator node $D$ has a variable value $\operatorname{dist}(v)=0$. The starting value of the variable is $\operatorname{pred}(v)=0$ which means that the node is not a router.

The procedure $B S(G, d)$ ensures that all nodes $v \in V$ are tested, each variable of the node $\operatorname{dist}(v)$ is evaluated accordingly and the value is equal to the length of path from the node $V$ to the node $D$. As the validation data, the queue $Q$ combined with the function First_Out(Q) (exclude the first element of the queue) and Insert_End(Q) (insert rows at the end of an element) is used.

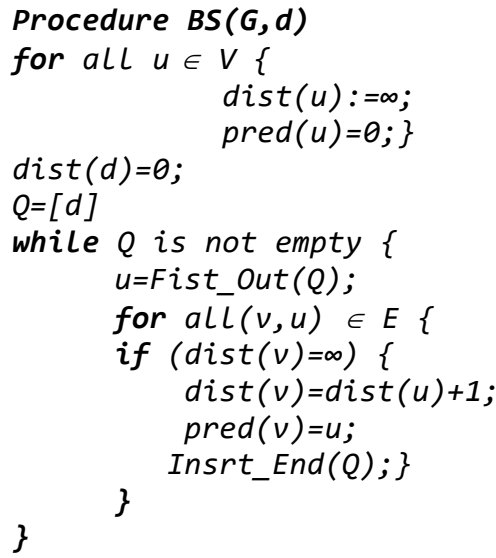

By completion of a cycle, the variable $\operatorname{dist}(v)$ has the value of the minimum distance from nodes $V$ to $D$; however, values of $\operatorname{pred}(v)$ allow creating a path from any peak $V$ to the coordinator node $D$. Appropriately, the given approach can be considered optimal in tasks, which require graph path metrics.

Using the mathematical model of the network that has been defined in article [3] written by the other author, it is necessary to introduce an approach that will list and take into account the remaining amount of power in each of the network nodes. It is certain that the remaining amount of energy in the node is a positive figure. In this case, it is proposed to use the Dijkstra's algorithm for the graph analysis provided that $l(u, v) \geq 0$ where $l(u, v)=E_{0}-E_{n}$. Thus, it is necessary to build the road through the paths with minimum energy difference coefficient.

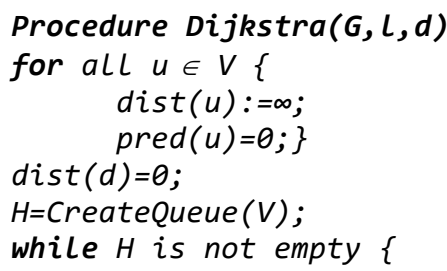

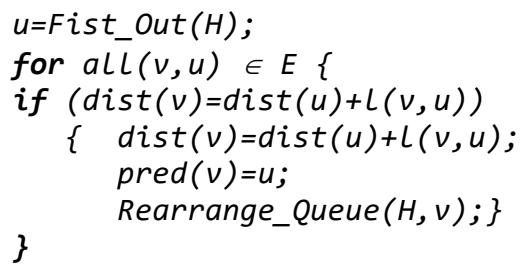

The significant difference in the procedure is the use of priority queue $H$ where all the elements are sorted by using $\operatorname{dist}(v)$ values. The procedure CreateQueue() forms the priority queue $H$ placing the coordinator node $\operatorname{dist}(d)=0$ at the beginning of the queue. Procedure Rearrange_Queue $(H, v)$ provides the reconfiguration of the queue in the case of any changes in metrics $V$.

Every time the cycle is executed, two conditions are completed:

1. There is a $d>0$ value where all nodes on display are $\operatorname{dist}(v) \leq d$ and the remaining queue nodes are $\operatorname{dist}(v) \geq d$.

2. For each node $V$ in the queue, the value $\operatorname{dist}(v)$ is equal to $\infty$ or with the minimal length of the path from $V$ to $D$. In addition, the unused nodes are removed from the queue.

The fulfillment of these conditions guarantees the optimal solution to the algorithm.

If there is a need to assess the changes in the metrics graph, it is possible to use the Bellman-Ford algorithm. When the algorithm is in use with the starting value of metrics, the node $V$ may change frequently. Every metric changes are implemented through the following procedure:

$\operatorname{Update} M_{-}(v, u): \operatorname{dist}(v)-\min \{\operatorname{dist}(v), \operatorname{dist}(u), l(v, u)\}$

By completing the procedure, certain properties are engaged:

- The usage of the procedure does not increase the $\operatorname{dist}(v)$ value;

- The optimum result corresponds to the minimal dist $(v)$ value, which means that regular usage of procedure Update_M will not be of negative effect.

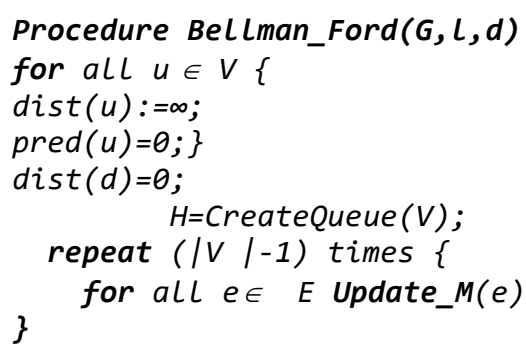

For the described algorithms to be used in wireless sensor networks, a slight adaption of each algorithm is necessary. To improve the Dijkstra's algorithm, it is proposed to introduce an additional function Best_Value $(Q)$ that allows distributing the node $U$ from the cluster $Q$ with the minimum path metrics to the node $D$. The entire set of nodes $V$ is considered as not 
One of the authors [6] offers to ensure the relocation of the coordinator via a displayed network perimeter. This approach is based on the fact that whilst the network is functioning the elements that are located in the centre contain a lower amount of the remaining power in the nodes.

Basagni [1] proposes the usage of MRE (Maximum Residual Energy) for the algorithm of coordinator movement. When the coordinator role selection method uses the GMRE algorithm, it defines the remaining amount of energy in the adjacent nodes and chooses one of the nodes with the largest amount of energy left in it. In this case, the planning of the optimal motion of the coordinator is pegged to searching the maximum amount of the remaining energy in the nodes.

There are some definitions for the function $E_{k}$ that allows determining the location of the coordinator:

- $\quad E_{k}(t)=\frac{1}{|D(k)|} \sum_{j \in D(k)} E_{r j}(t) ;$

- $E_{k}(t)=\min _{j \in D(k)} E_{r j}(t)$;

- $E_{k}(t)=\max _{j \in D(k)} E_{r j}(t)$.

Regardless of how the function $E_{k}$ is defined, the remaining amount of energy characterises the overall state of the network only partially and cannot be used as the sole condition. For example, a node with the smallest amount of remaining energy $E_{0}$ and the lowest power consumption can work for a longer period of time than a node with the greatest $E_{0}$ and the greatest energy consumption. The use of MRE method simply assigns a coordinator role to the node with the highest $E_{0}$ value; in case the network agents do not contribute to any new topology changes, this method will not increase the overall network life expectancy.

The article offers an alternative method: choosing a location $p_{n}$ for the virtual coordinator in every step of the operation that allows the network to function for as long as possible on the condition that the network topology is not changed. The name of the energy method MREML (Maximum Residual Energy Maximal Lifetime) can be defined by using an analogue:

$$
E_{k}(t)=\arg \left(\max _{k \in S\left(p_{n}\right)} \min _{n \in D(k)} \frac{E_{0}^{n}(t)}{p_{k}^{n}(t)}\right),
$$

where $p_{k}^{n}$ is all the consumed energy in one cycle of operation in the node $K$.

If the time the coordinator spends in each of the node positions is equal to $t_{k}$ and the remaining amount of energy prior to placing the coordinator in the position $E_{0}^{n}(t)$ is known as well as the amount of the energy in the node when the coordinator leaves the position is $E_{0}^{n}\left(t+t_{k}\right)$, then the consumed amount of energy by each node can be stated by the following formula:

$$
p_{k}^{n}=\frac{E_{0}^{n}(t)-E_{0}^{n}\left(t+t_{k}\right)}{t_{k}}
$$

The difference between MRE and MREML methods is that the former uses the local information of the network nodes regarding the remaining amount of energy that constantly requires additional power in order to analyse the remaining amount of energy in the adjacent nodes. The method proposed in the article uses network agents and the static coordinator node for storing information. In case of any changes in the network topology, this approach allows obtaining a global overview of the entire network configuration as well as the virtual coordinator node is able to occupy the best position in order to collect information from the nodes.

\section{CONCLUSION}

The article has described a coordinator role mobility method, which allows increasing the life expectancy of a wireless sensor network evening out the remaining amount of energy among all the network elements.

In the network scenarios with the ability to modify the routing protocol, the use of modified Bellman-Ford and Dijkstra's algorithms is proposed for maintaining an optimal data flow route based on the increase of the criteria of network life expectancy. The remaining amount of energy is defined as the most significant criteria for the choice of a path within a certain network node in the stage of iteration.

To avoid unnecessary network topology changes, particular conditions that determine the moment of the network structural changes are indicated in the article. Primary conditions for topology changes have been asserted based on technical data obtained from the sensor nodes and network structure analysis during the operation of the wireless sensor network:

- The condition which depends on the location of the node;

- The condition which depends on the operating segment of the network coordinator;

- The condition which depends on the unbalanced consumed energy in the network nodes.

Using the descriptions of the calculation method for the life expectancy of the wireless sensor network nodes indicated in other articles [3], the condition that is related to the amount of energy remaining in the nodes has been proposed to be integrated on the routing protocol level.

As a result, the article proposes the algorithm for a wireless sensor network topology change that uses the coordinator mobility role of the network as the primary factor for the topology change in the network.

To reduce the amount of energy consumed in the nodes, the article proposes a method that anticipates the implementation of an independent software module of the network, which will control the state of each network node and promote the change of the network topology regardless of the form of action of the node. Accordingly, a low-power wireless set will be used as a means of communication, which will transmit technical information to the network agent manager.

In some cases, there may be situations where the physical coordinator node cannot be reached or it may be impossible to establish a communication route between the sensor node and the coordinator; the alternative will be to use the virtual coordinator, which is managed by using heuristic algorithms. 


\section{ACKNOWLEDGEMENT}

The research has partly been funded by the Latvian Council of Science within Project Z12.0342 "Development of Models and Methods Based on Distributed Artificial Intelligence, Knowledge Management and Advanced Web Technologies for Applied Intelligent Software”.

\section{REFERENCES}

[1] S. Basagni, A. Carosi, and C. Petrioli, "Controlled Vs. Uncontrolled Mobility in Wireless Sensor Networks: Some Performance Insights," in 2007 IEEE 66th Vehicular Technology Conference, Sep. 2007. pp. 269273. https://doi.org/10.1109/vetecf.2007.70

[2] D. M. Blough and P. Santi, "Investigating upper bounds on network lifetime extension for cell-based energy conservation techniques in stationary ad hoc networks," in Proceedings of the 8th annual international conference on Mobile computing and networking MobiCom '02, 2002. pp. 183-192. https://doi.org/10.1145/570665.570668

[3] A. Jurenoks and L. Novickis, "Wireless sensor networks lifetime assessment model development," Environment. Technology. Resources. Proceedings of the International Scientific and Practical Conference, vol. 3, Jun. 2015. pp. 121-126. https://doi.org/10.17770/etr2015vol3.508

[4] H. Karl and A. Willig, Protocols and Architecture for Wireless Sensor Networks. Chichester: John Wiley \& Sons, 2005. https://doi.org/10.1002/0470095121

[5] S. Kumar, A. Arora, and T. H. Lai, "On the lifetime analysis of alwayson wireless sensor network applications," in IEEE International Conference on Mobile Adhoc and Sensor Systems Conference, 2005. p. 3. https://doi.org/10.1109/mahss.2005.1542797

[6] R. Mautz and S. Tilch, "Survey of optical indoor positioning systems," in 2011 International Conference on Indoor Positioning and Indoor Navigation, $\quad$ Sep. $2011 . \quad$ pp. $1-7$. https://doi.org/10.1109/ipin.2011.6071925

[7] G. G. Messier and I. G. Finvers, "Traffic models for medical wireless sensor networks," IEEE Communications Letters, vol. 11, no. 1, pp. 1315, Jan. 2007. https://doi.org/10.1109/lcomm.2007.061291.
[8] R. Moghe, Y. Yang, F. Lambert, and D. Divan, "Design of a low cost self powered "Stick-on" current and temperature wireless sensor for utility assets," in 2010 IEEE Energy Conversion Congress and $\begin{array}{llll}\text { Exposition, } & \text { Sep. } & 2010 . & \text { pp. }\end{array}$ https://doi.org/10.1109/ecce.2010.5618430

[9] V. Raghunathan, C. Schurgers, Sung Park, and M. B. Srivastava, "Energy-aware wireless microsensor networks," IEEE Signal Processing Magazine, vol. 19, no. 2, pp. 40-50, Mar. 2002. https://doi.org/10.1109/79.985679

[10] A. N. Vabiŝevič, "Opredelenie položenija v prostranstve elementov besprovodnoj sensornoj seti s pomosju inercialnyh sensorov," in Tezisy dokladov naučno-tehničeskoj konferencii studentov, aspirantov $i$ molodyh specialistov, 2010. pp. 151-152.

[11] M. V. Gekk, T. E. Istomin, Ja. R. Fajzulhakov, and A. V. Čečendaev, "Adaptivnyj algoritm bystroj dostavki soobsenij po vydelennym napravlenijam dlja besprovodnyh setej datčikov," Vestnik molodyh učenyh "Lomonosov". Vypusk III, 2006. pp. 55-60.

Aleksejs Jurenoks is an Assistant Professor at Riga Technical University. He is the author of 21 publications and 6 educational books. His research interests include adaptive systems, intelligent systems and automation. He is regularly involved in different EU-funded projects: eLOGMAR-M (FP6, 2004-2006); IST4Balt (FP6, 2004-2007), UNITE (FP6, 2006-2008) and BONITA (INTERREG, 2008-2012). He is a member of the program committee for the CISTI Iberian Conference on Information Systems and Technologies. E-mail: aleksejs.jurenoks@rtu.lv

Dejan Jokićb is the founder of the annual summer school at the University of East Sarajevo which he has collaborated with colleagues Prof. Slobodan Lubara and Ms. Milica Ristovic. He received his Doctoral degree from the University of East Sarajevo. His research interests include automation, robotics and mechatronic systems. He specialises in the design of FPGA programmable logic control of robotics. Furthermore, he was a member of the program committee for the 2016 IFAC/IEEE Conference on Programmable Devices and Embedded Systems.

E-mail: dejan.jokic@etf.unssa.rs.ba 